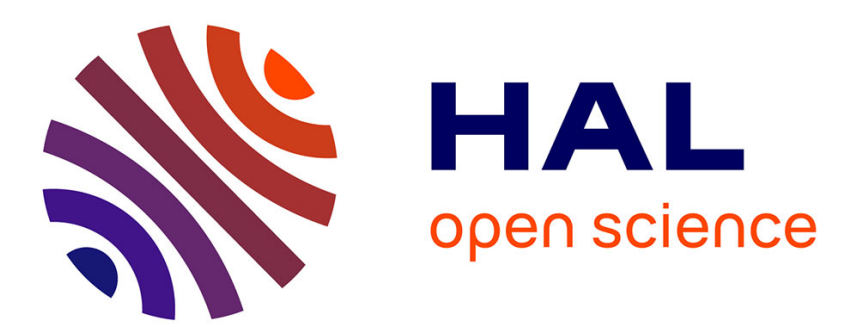

\title{
Influence of gait speed on the control of mediolateral dynamic stability during gait initiation.
}

Teddy Caderby, Eric Yiou, Nicolas Peyrot, Mickaël Begon, Georges Dalleau

\section{To cite this version:}

Teddy Caderby, Eric Yiou, Nicolas Peyrot, Mickaël Begon, Georges Dalleau. Influence of gait speed on the control of mediolateral dynamic stability during gait initiation.. Journal of Biomechanics, 2014, 47 (2), pp.417-423. 10.1016/j.jbiomech.2013.11.011 . hal-00925400v2

\section{HAL Id: hal-00925400 \\ https://hal.science/hal-00925400v2}

Submitted on 28 Oct 2016

HAL is a multi-disciplinary open access archive for the deposit and dissemination of scientific research documents, whether they are published or not. The documents may come from teaching and research institutions in France or abroad, or from public or private research centers.
L'archive ouverte pluridisciplinaire HAL, est destinée au dépôt et à la diffusion de documents scientifiques de niveau recherche, publiés ou non, émanant des établissements d'enseignement et de recherche français ou étrangers, des laboratoires publics ou privés. 


\title{
Influence of gait speed on the control of mediolateral dynamic stability during gait initiation
}

\author{
Teddy Caderby ${ }^{\mathrm{a}, *}$, Eric Yiou $^{\mathrm{b}}$, Nicolas Peyrot $^{\mathrm{a}}$, Mickaël Begon $^{\mathrm{c}}$, Georges Dalleau $^{\mathrm{a}}$ \\ a Laboratoire DIMPS-CURAPS, UFR des Sciences de l'Homme et de l'Environnement, Université de la Réunion, 117 rue du Général Ailleret, \\ 97430 Le Tampon, Ile de la Réunion \\ b Laboratoire CIAMS, Team RIME, UFR STAPS, Université Paris-Sud, 91440 Orsay Cedex, France \\ ' Laboratoire de Simulation et Modélisation du Mouvement, Département de Kinésiologie, Université de Montréal, Laval, QC, Canada
}

\begin{abstract}
A B S T R A C T
This study investigated the influence of gait speed on the control of mediolateral dynamic stability during gait initiation. Thirteen healthy young adults initiated gait at three self-selected speeds: Slow, Normal and Fast. The results indicated that the duration of anticipatory postural adjustments (APA) decreased from Slow to Fast, i.e. the time allocated to propel the centre of mass (COM) towards the stance-leg side was shortened. Likely as an attempt at compensation, the peak of the anticipatory centre of pressure (COP) shift increased. However, COP compensation was not fully efficient since the results indicated that the mediolateral COM shift towards the stance-leg side at swing foot-off decreased with gait speed. Consequently, the COM shift towards the swing-leg side at swing heel-contact increased from Slow to Fast, indicating that the mediolateral COM fall during step execution increased as gait speed rose. However, this increased COM fall was compensated by greater step width so that the margin of stability (the distance between the base-of-support boundary and the mediolateral component of the "extrapolated centre of mass") at heel-contact remained unchanged across the speed conditions. Furthermore, a positive correlation between the mediolateral extrapolated COM position at heel-contact and step width was found, indicating that the greater the mediolateral COM fall, the greater the step width. Globally, these results suggest that mediolateral APA and step width are modulated with gait speed so as to maintain equivalent mediolateral dynamical stability at the time of swing heel-contact.
\end{abstract}

\section{Introduction}

Gait initiation (GI), corresponding to the transition from stationary standing to walking, is a functional task that is commonly performed in daily life. As emphasised in the literature, this task provides a challenge to dynamic stability, especially in the mediolateral (ML) direction (Lyon and Day, 1997; Mcllroy and Maki, 1999). Indeed, the act of lifting the swing foot to execute the first step induces a reduction of the size of the base of support (BOS), which is then limited to stance-foot contact with the ground. It follows that if no action on the centre of mass (COM) is undertaken before the time of swing foot-off, i.e. if the COM is not moved above the stance foot, the whole-body will tend to fall laterally towards the swing-leg side during step execution, potentially causing a loss of balance and a sideways fall.

\footnotetext{
* Corresponding author. Tel.: +2622625795 91; fax: +262262579571.

E-mail addresses: teddy.caderby@univ-reunion.fr, caderby.t@hotmail.fr (T. Caderby).
}

It is known that centrally-initiated dynamic phenomena, termed "anticipatory postural adjustments" (APA), precede the onset of voluntary movement. These APA aimed to stabilise the posture or assist the motor performance (Bouisset and Do, 2008; Yiou et al., 2012a). APA are observed before the step execution (beginning at swing heel-off) during GI. Along the ML direction, these APA are manifested as a centre of pressure (COP) shift towards the swing-leg side that propels the COM towards the stance-leg side prior to swing foot-off (Mcllroy and Maki, 1999; Rogers et al., 2001; Yiou and Do, 2011). Although they do not directly propel the COM above the stance foot at foot-off (Jian et al., 1993), ML APA reduce the extent to which the COM falls toward the swing-leg side during step execution. ML APA thus constitute a crucial mechanism for controlling ML stability during GI (Mcllroy and Maki, 1999). It is noteworthy that ML stability during GI may also be controlled via ML swing-foot placement at swing heel-contact (Lyon and Day, 1997; Zettel et al., 2002a, 2002b). By regulating the ML swing-foot placement (i.e. step width), individuals may maintain the COM within the BOS and thus ensure ML stability.

APA have also been described along the anteroposterior direction during GI. These APA are manifested as a backward COP shift 
that generates the initial propulsive forces necessary to reach the intended gait speed at the end of the first step (Brenière et al., 1987). The influence of gait speed on these APA has been extensively investigated (e.g. Brenière et al., 1987; Ito et al., 2003; Lepers and Brenière, 1995). In contrast, the question how the ML stabilizing features (including ML APA and ML foot placement) and related ML stability are modulated with gait speed is far less documented. To our knowledge, only one recent study has examined the influence of speed on ML dynamic stability control during volitional stepping (Singer et al., 2013). However, this study focused mainly on ML stability control during the phase of step termination (termed the "restabilisation" phase) and thus did not clearly investigate the speed effect on this control during the step initiation phase.

Increasing gait speed amplifies the accelerations acting on the body (Menz et al., 2003; Shkuratova et al., 2004), which may consequently result in a greater challenge to ML dynamic stability during GI. Interestingly, recent studies on rapid leg flexion showed that young healthy participants were able to modulate ML APA in order to maintain ML dynamic stability unchanged in situations with a postural constraint, e.g. temporal pressure (Yiou et al., 2012b) or elevated support surface (Yiou et al., 2011). Similarly, previous studies showed that, during reactive stepping initiation, participants used a strategy of lateral swing foot placement, along with the inclusion of larger ML APA, to compensate for postural perturbation induced by force-plate translation (e.g. Zettel et al., 2002a, 2002b). These findings suggest that, when facing a postural constraint, the central nervous system (CNS) has the capacity to modulate ML APA and step width in order to maintain equivalent dynamic stability.

The present study investigated the influence of gait speed on ML dynamic stability control during GI. We hypothesised that healthy young adults modulate the temporo-spatial features of ML APA and ML foot placement as gait speed increases so as to maintain equivalent ML dynamic stability at the time of swing heel-contact.

\section{Methods}

\subsection{Subjects}

Thirteen healthy subjects ( 6 males, 7 females; age: $27 \pm 6$ years, height: $171+9 \mathrm{~cm}$, body mass: $68+10 \mathrm{~kg}$, body mass index: $23+2 \mathrm{~kg} / \mathrm{m}^{2}$ ) participated in this experiment. All gave written consent after being fully informed of the test procedure, which was approved by the local ethics committee.

\subsection{Experimental set-up and procedure}

Gait was initiated from a force-plate $(46.4 \times 50.8 \mathrm{~cm}$, AMTI, USA) located at the beginning of a 5-m walkway (Fig. 1). A larger force-plate $(90 \times 90 \mathrm{~cm}$, AMTI, USA) was located immediately in front of this initial force-plate so that the first step landed onto it. The two force-plates, embedded in the walkway, recorded the ground reaction forces and moments. Reflective skin markers (9-mm diameter) were placed bilaterally at the hallux (toe marker), head of the fifth metatarsus and posterior calcaneus (heel marker). A five-camera motion capture system (Vicon MX-T40, Oxford, UK) with 64 analog channels was used to collect simultaneously the kinematic data at $200 \mathrm{~Hz}$ and the force-plate data at $1000 \mathrm{~Hz}$.

Initially, subjects stood barefoot in a natural upright posture with their arms alongside their trunk. They were instructed to stand as still as possible with their body weight distributed evenly between their legs. Gaze was fixed on a $10-\mathrm{cm}$ diameter target placed at eye level and $6 \mathrm{~m}$ distant. After receiving a verbal "all set" signal, subjects initiated gait on their own initiative and continued walking straight ahead to the end of the walkway. Subjects chose their natural swing leg and maintained it throughout the experiment. After each trial, they had to reposition themselves in the same standardized feet position (see McIlroy and Maki, 1997) previously marked on the first force-plate. The experimenter triggered data acquisition when the subject was motionless and at least $1 \mathrm{~s}$ before the "all set" signal.

Gait initiation was performed under three speed conditions: natural pace (Normal condition), slower-than-natural pace (Slow) and as quickly as possible
(Fast). The order of conditions was randomized across the subjects. In each speed condition, subjects performed two familiarisation trials and then five trials were collected. Subjects rested for 2 min between the speed conditions.

\subsection{Data analysis}

Kinematic and force-plate data were low-pass filtered using a Butterworth filter with a $15 \mathrm{~Hz}$ (Mickelborough et al., 2000) and a $10 \mathrm{~Hz}$ (Corbeil and Anaka, 2011) cut-off frequency, respectively. The ML coordinate of the COP was computed from force-plate data in accordance with the manufacturer's instructions (AMTI Manual). Formula is given in Appendix A.

Instantaneous ML acceleration of the COM ( $\left.\mathrm{y}^{\prime \prime} \mathrm{COM}\right)$ was determined from the ML ground reaction force according to Newton's second law. ML COM velocity and displacement were computed by successive numerical integration of the COM acceleration (Brenière et al., 1987). By convention, COM displacement and velocity and COP displacement were considered positive when directed toward the swingleg side.

The following instants were determined on the biomechanical traces (Fig. 2): Gl onset $\left(t_{0}\right)$, swing heel-off (HO), swing foot-off (FO) and swing heel-contact (HC) Time $t_{0}$ corresponded to the instant when the $\mathrm{y}^{\prime \prime} \mathrm{COM}$ trace deviated 2.5 standard deviations from its baseline value (Yiou et al., 2012b). Heel-off and foot-off corresponded to the instants when the vertical position of the heel marker and the anterior position of the toe marker increased respectively by $3 \mathrm{~mm}$ from their position in the initial static posture. Heel-contact corresponded to the instant when the vertical ground reaction force measured by the second force-plate exceeded $10 \mathrm{~N}$ (Ghoussayni et al., 2004).

\subsection{Dependant variables}

Gait initiation was divided into three phases: APA (from $t_{0}$ to HO), foot lift (from $\mathrm{HO}$ to FO) and step execution (from FO to HC). The duration of each phase was reported. APA amplitude was characterised with the peak of lateral COP shift toward the swing-leg side. ML COM velocity and displacement at heel-off, foot-off and heel-contact were calculated. The peak of anteroposterior COM velocity was calculated to quantify gait speed (Brenière and Do, 1986; Caderby et al., 2013). The ML COM position in the initial upright posture was estimated by averaging the ML COP position during the 250-ms period preceding the "all set" signal (Mcllroy and Maki, 1999).

An adaptation of the "margin of stability" (MOS) introduced by Hof et al. (2005) was used to quantify ML dynamic stability at heel-contact. In the present study, the MOS corresponded to the difference between the ML boundary of the BOS $\left(B O S_{\text {max }}\right)$ and the ML position of the "extrapolated centre of mass" at heelcontact $\left(\mathrm{YcoM}_{\mathrm{HC}}\right)$, i.e. $\mathrm{MOS}=\mathrm{BOS}_{\mathrm{ymax}}-\mathrm{YcoM}_{\mathrm{HC}}$. Because kinematic data showed that the swing foot-strike was systematically made with the heel, BOS $_{\text {ymax }}$ was estimated with the ML position of the heel marker of the swing foot at heelcontact. The ML distance between the position of the swing heel marker at heelcontact and the position of the stance heel marker at $t_{0}$ represented the step width, and was representative of the size of the ML BOS.

Based on the study of Hof et al. (2005), the ML position of the extrapolated $\mathrm{COM}$ at heel-contact $\left(\mathrm{YcoM}_{\mathrm{HC}}\right)$ was calculated as follows:

$\mathrm{YcoM}_{\mathrm{HC}}=\mathrm{yCOM}_{\mathrm{HC}}+\frac{\mathrm{y}^{\prime} \mathrm{COM}_{\mathrm{HC}}}{\omega_{0}}$,

where $\mathrm{yCOM}_{\mathrm{HC}}$ and $\mathrm{y}^{\prime} \mathrm{COM}_{\mathrm{HC}}$ are respectively the ML COM position and velocity at heel-contact, and $\omega_{0}$ is the eigenfrequency of the body modelled as an inverted pendulum calculated as:

$\omega_{0}=\sqrt{\frac{g}{l}}$

where $g=9.81 \mathrm{~m} / \mathrm{s}^{2}$ is the gravitational acceleration and $l$ is the length of the inverted pendulum, which in this study corresponded to $57.5 \%$ of the body height (Winter, 1990).

ML dynamic stability at heel-contact is ensured on the condition that $\mathrm{YcoM}_{\mathrm{HC}}$ is within BOS $_{\mathrm{ymax}}$, which corresponds to a positive MOS. A negative MOS indicates ML instability and implies that a corrective action (e.g. in the form of an additional lateral step) has to be undertaken to maintain balance.

\subsection{Statistical analysis}

Mean values and standard deviations of the dependant variables were calculated for each gait speed condition. Repeated-measures ANOVA with gait speed as a factor were conducted separately on these variables. Tukey post hoc analysis was performed when a statistical difference was found. Pearson's correlation coefficient $(r)$ was used to determine the relationship between the variables. The level of statistical significance was set at $\alpha=0.05$. 


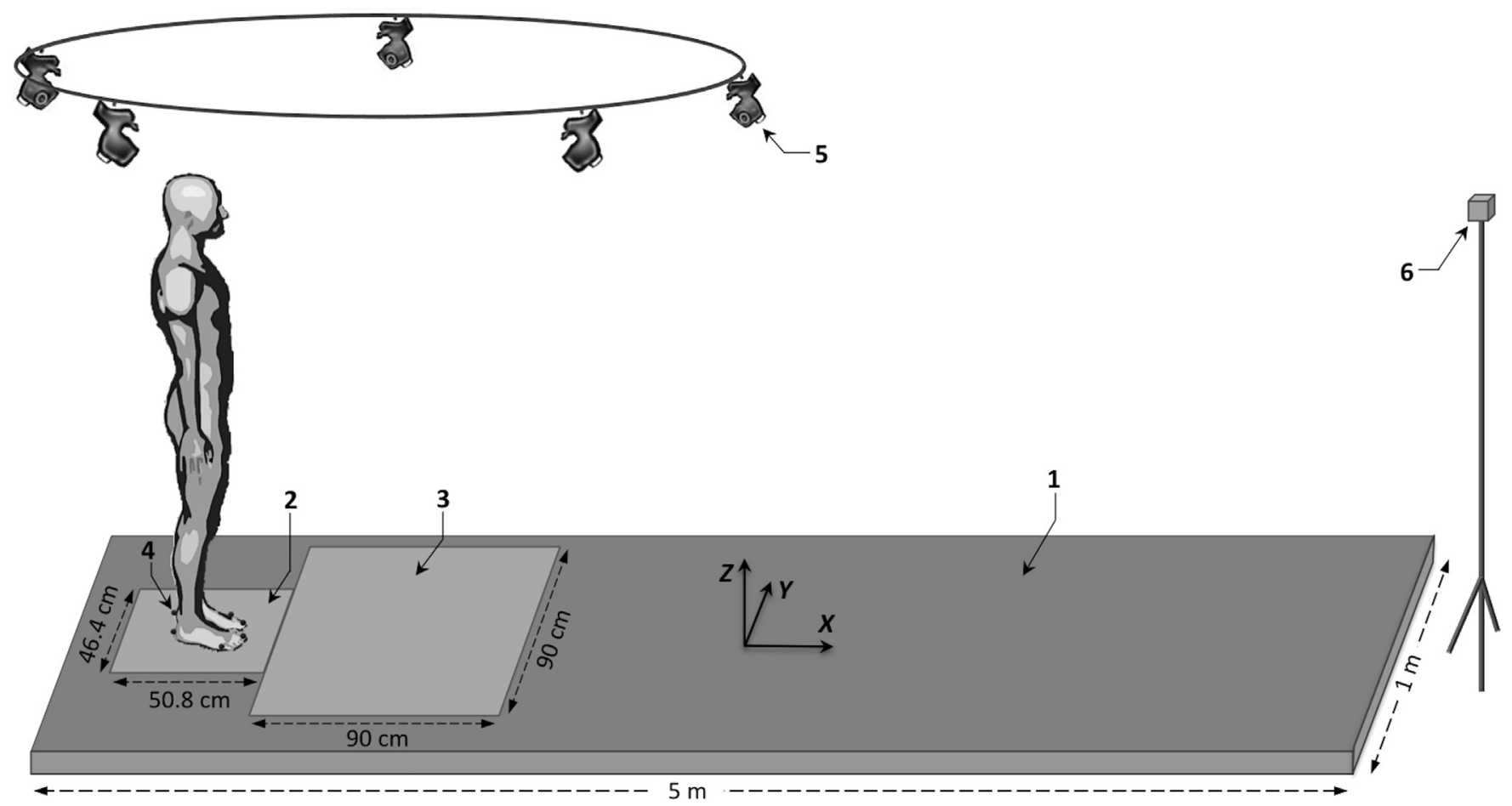

Fig. 1. Schematic illustration of the experimental set-up. Legend: (1) walkway; (2) and (3) force-plates; (4) reflective marker; (5) Vicon camera; and (6) visual target.

\section{Results}

\subsection{Description of the biomechanical traces}

The time course of the biomechanical traces was very similar in the three speed conditions (Fig. 2). Swing heel-off was systematically preceded by postural dynamics corresponding to APA. During APA, COP displacement reached a peak value towards the swing leg, while the COM displacement and velocity were directed toward the stance leg. The COM velocity trace reached the first peak value towards the stance-leg side at around heel-off. This trace then dropped towards the swing-leg side and the second peak value towards this side was reached a few milliseconds after heel-contact. The COM displacement reached a peak value toward the stance-leg side during the execution phase. The COM then fell towards the swing-leg side. Anteroposterior COM velocity increased progressively until it reached a peak value a few milliseconds after heel-contact.

\subsection{Gait speed}

As expected, a significant effect of the condition was found for gait speed $(p<0.001)$. Post hoc tests showed that this parameter increased significantly from Slow to Fast $(p<0.001)$. Gait speed was $0.75 \pm 0.11 \mathrm{~m} / \mathrm{s}$ in Slow, $1.08 \pm 0.19 \mathrm{~m} / \mathrm{s}$ in Normal and $1.51 \pm 0.26 \mathrm{~m} / \mathrm{s}$ in Fast.

\subsection{Influence of gait speed on postural parameters}

The initial ML COM position during quiet standing did not significantly change across gait speed conditions $(p>0.05)$. In contrast, a significant speed effect was found for APA duration $(p<0.001)$, foot lift duration $(p<0.001)$, and step execution duration $(p<0.001)$. Post hoc tests indicated that the duration of each of these phases significantly decreased as gait speed increased (Fig. 3).
In regards to the spatial components of GI, the ANOVA revealed that ML COM velocity measured at heel-off, foot-off and heelcontact did not change significantly with gait speed $(p>0.05)$. In contrast, there was a significant speed effect on the following variables: peak of ML COP shift during APA $(p<0.001)$, ML COM displacement at heel-off ( $p<0.001)$, foot-off $(p<0.001)$ and heelcontact $(p<0.001)$. Specifically, post hoc tests indicated that the peak of ML COP shift towards the swing-leg side increased significantly with gait speed, while the ML COM displacement toward the stance-leg side at heel-off and foot-off decreased significantly with gait speed. Post hoc tests further showed that the ML COM displacement toward the swing-leg side at heelcontact increased significantly with gait speed (Fig. 4).

Under all speed conditions, the ML component of the extrapolated COM $\left(\mathrm{YcoM}_{\mathrm{HC}}\right)$ was located within the ML BOS at heelcontact. However, the results indicated that $\mathrm{YcoM}_{\mathrm{HC}}$ was located further toward the swing-leg side in Fast compared to Slow and Normal speeds (Fig. 5). The ANOVA confirmed this observation and revealed a significant effect of speed on $\operatorname{YcoM}_{\mathrm{HC}}(p<0.01)$. A significant speed effect was also found for step width $(p<0.01)$. Post hoc tests further showed that both $\mathrm{YcoM}_{\mathrm{HC}}$ and step width reached a significantly higher value in Fast than in Slow and Normal speeds (Fig. 5). There was no statistical difference between Slow and Normal for these two parameters. Despite these significant changes in $\mathrm{YcoM}_{\mathrm{HC}}$ and step width, gait speed had no significant effect on MOS $(p>0.05)$. Finally, a significant correlation was found between $\mathrm{YcoM}_{\mathrm{HC}}$ and step width $(r=0.87$, $p<0.001$, Fig. 6). In contrast, MOS and $\mathrm{YcoM}_{\mathrm{HC}}$ were not significantly correlated $(p>0.05)$

\section{Discussion}

To our knowledge, this is the first study to investigate the influence of gait speed on ML stability control during GI. Previous studies investigating the speed effect on the GI process focused exclusively on sagittal plane motion (among many others, see 

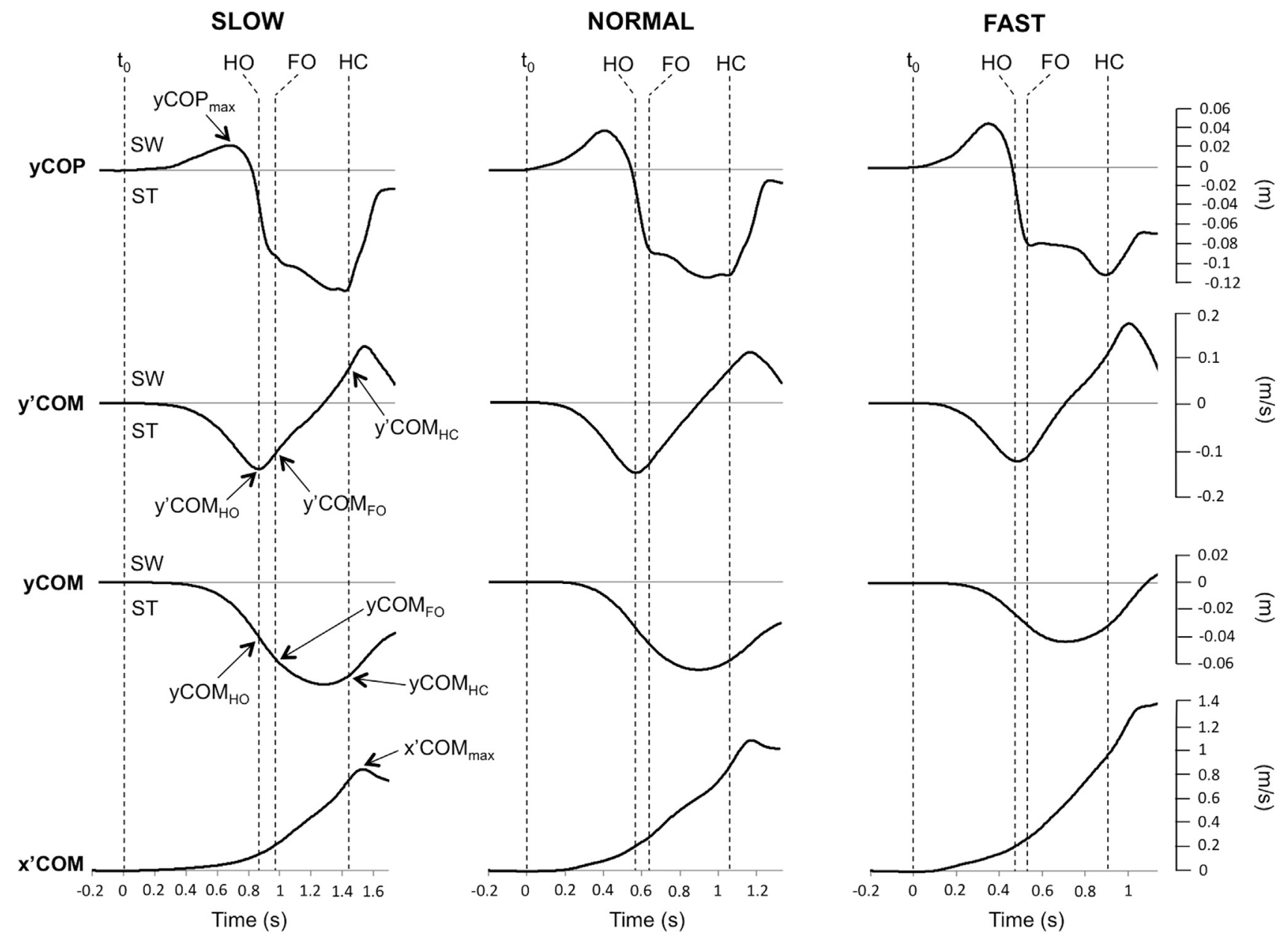

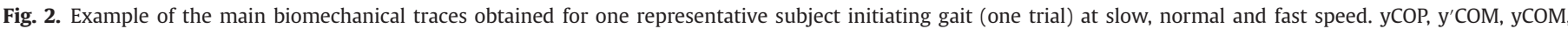

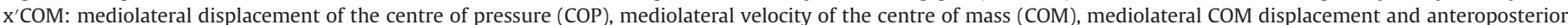

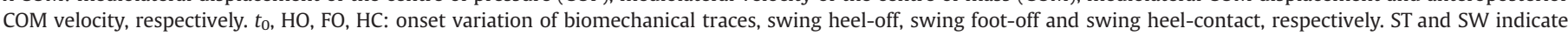

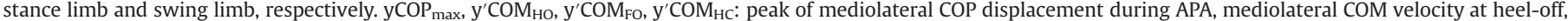

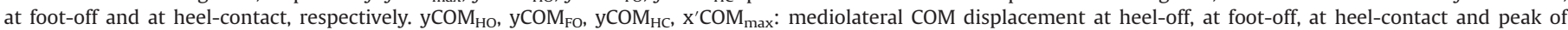
anteroposterior COM velocity at the end of the first step, respectively.

Brenière et al., 1987; Lepers and Brenière, 1995). These studies globally reported that the amplitude (in term of peak backward COP shift during APA) and duration of APA along the anteroposterior axis increased with gait speed in order to generate higher forward COM propulsion. In the ML direction, our results showed that the peak of ML COP displacement during APA (i.e. ML APA amplitude) also increased with gait speed. Surprisingly, ML APA duration decreased with gait speed, which contrasts with the previous findings on the APA duration along anteroposterior axis. Because the end of the APA phase was similarly defined in these other studies and the present study (heel-off instant), this observation suggests that APA onset during GI is directionally dependant, a finding previously mentioned by Lin and Yang (2011). This direction dependence of APA onset may underline the neuromuscular system's ability to independently create the initial conditions for both forward progression and ML stability, probably in order to achieve the safest and most efficient stepping strategy. Nonetheless, as the APA parameters along the anteroposterior and mediolateral directions vary according to gait speed, it is possible that the CNS exerts a global control of the anticipatory postural dynamics in the horizontal plane.

Based on the inverted pendulum model, previous studies showed that the COP shift during APA serves to proportionally accelerate the COM in the opposite direction (Brenière et al., 1987; Polcyn et al., 1998; Winter, 1995). In the present study, the increase in the ML COP shift with increased gait speed would therefore $a$ priori be expected to be matched by an increase in the initial COM dynamics at the end of APA. However, contrasting with this expectation, our results showed that the COM displacement toward the stance-leg side at both heel-off and foot-off instants decreased as gait speed increased, while ML COM velocity at these two instants did not change. Therefore, the efficiency of ML APA to propel the COM towards the stance-leg side seems altered as gait speed increases. Similar results regarding COP and COM dynamics during APA have been reported in recent studies (Corbeil and Anaka, 2011; Singer et al., 2013), but the authors did not provide experimental data to explain these findings. The result that the initial ML location of COM did not change across speed conditions suggests that these APA modifications could not be attributed to a change in body weight distribution during the initial posture (see Azuma et al., 2007). Rather, the attenuation of COM displacement toward the stance foot at heel-off and foot-off might be ascribed to the decrease in both APA and foot lift phase duration with gait speed ( $24 \%$ and $60 \%$ from Slow to Fast, respectively). Consequently, the time allocated to propelling the COM towards the stance-leg side was reduced. As argued below, additional compensations occurred later in the course of the GI process.

Using a mathematical model of the body falling freely under the action of gravity, Lyon and Day (1997) demonstrated that an attenuation of ML COM dynamics (displacement and velocity) at 

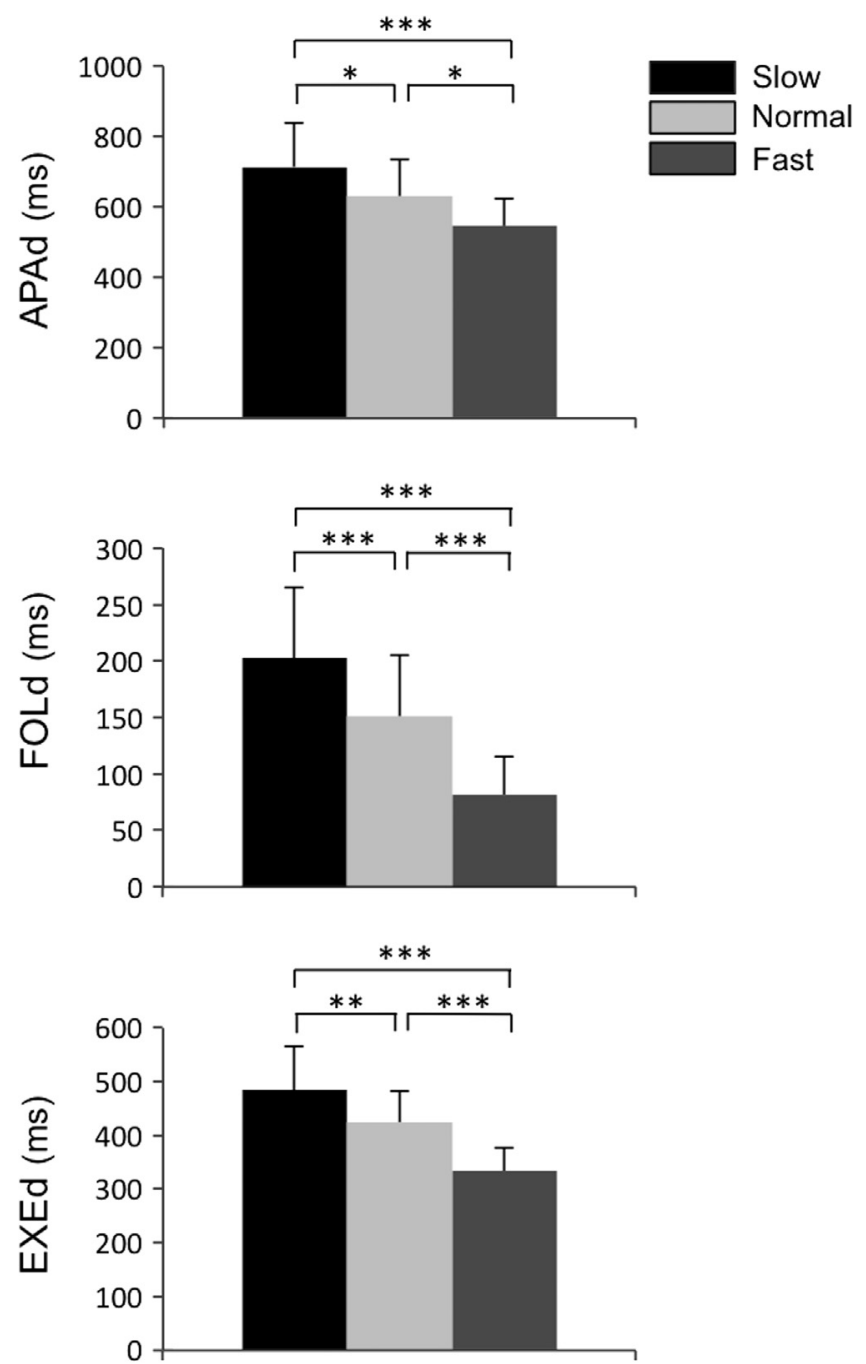

Fig. 3. Comparison of temporal parameters of gait initiation under the slow, normal and fast speed conditions. APAd, FOLd, EXEd: APA duration, foot lift duration and step execution duration, respectively. Reported values are means \pm 1 standard deviation (all subjects combined). *, ***, ***: significant difference with $p<0.05, p<0.01$ and $p<0.001$, respectively.

foot-off time resulted in a higher COM fall toward the swing-leg side during the subsequent step execution. The present result that the COM fall at heel-contact increased with gait speed (and with the related attenuation of the ML COM dynamics at foot-off) is therefore in line with this model. It is, however, noteworthy that this fall was minimised in the present study because the execution duration was concomitantly reduced with the increasing gait speed (31\% from Slow to Fast). As a direct consequence of this increase in the lateral $\mathrm{COM}$ fall, the extrapolated COM position at heel-contact $\left(\mathrm{YcoM}_{\mathrm{HC}}\right)$ was located further toward the swing-leg side in Fast compared to Slow and Normal. The difference of $\mathrm{YcoM}_{\mathrm{HC}}$ between Slow and Fast reached approximately $2 \mathrm{~cm}$. Therefore, if no action is undertaken on step width in Fast, the extrapolated COM position is expected to be located $2 \mathrm{~cm}$ closer to the BOS limits at heel-contact, thus reducing the margin of stability (MOS) by the same amount. In contrast, our results showed that step width increased by $2 \mathrm{~cm}$, resulting in an equivalent MOS value. This finding suggests that the participants were able to modulate their step width as a function of the extrapolated COM position (depending on ML APA and step execution duration) so as to maintain equivalent dynamical stability at heel-contact. Our results on correlation analyses strongly back this assumption. Indeed, we found that step width was highly correlated with $\mathrm{YcoM}_{\mathrm{HC}}(r=0.87)$, i.e. the greater the $\mathrm{YcoM}_{\mathrm{HC}}$
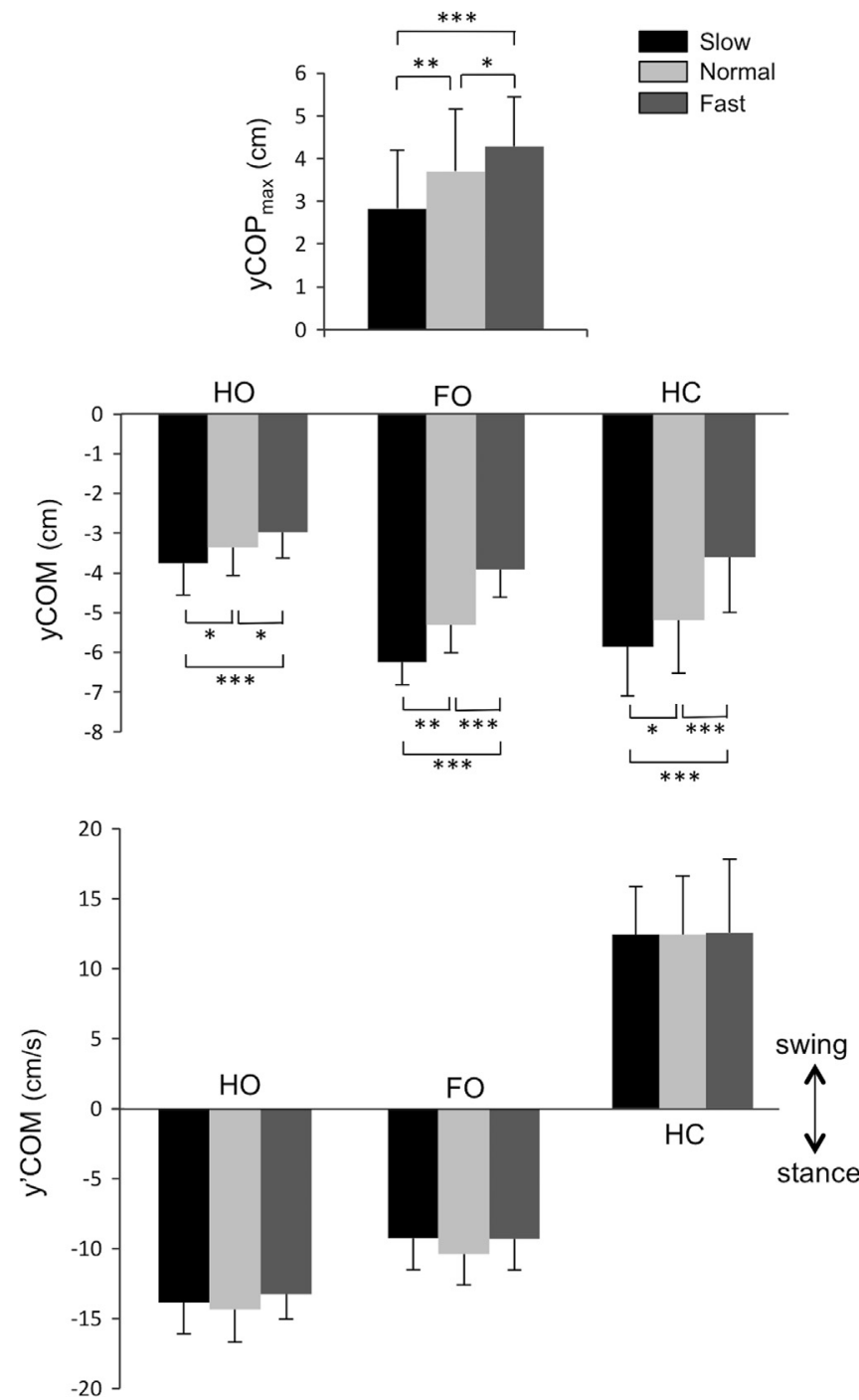

Fig. 4. Comparison of mediolateral APA amplitude and mediolateral centre of mass (COM) displacement and velocity at selected instants across the slow, normal and fast speed conditions. $\mathrm{yCOP}_{\max }, \mathrm{yCOM}, \mathrm{y}^{\prime} \mathrm{COM}$ : peak of lateral centre of pressure shift toward the swing leg during APA (APA amplitude), mediolateral COM displacement and mediolateral COM velocity, respectively. HO, FO, HC: swing heel-off, swing foot-off and swing heel-contact, respectively. Reported values are means \pm 1 standard deviation (all subjects combined). *, ***, ***: significant difference with $p<0.05, p<0.01$ and $p<0.001$, respectively.

location towards the swing-leg side, the greater the step width. Furthermore, step width varied with $\mathrm{YcoM}_{\mathrm{HC}}$ in such a way that MOS remained invariant. These findings are congruent with previous data on steady-state gait (Hof et al., 2007; Rosenblatt and Grabiner, 2010), which showed that MOS was not affected by gait speed in young healthy adults presumably due to an accurate regulation of ML foot placement. Therefore, taken globally, these results support our hypothesis and add to the growing evidence that the extrapolated COM position may function as a balance control parameter (e.g. Hasson et al., 2008; Yiou et al., 2011; Yiou et al., 2012b).

Interestingly, Singer et al. (2013) recently reported that the ML distance between the COM and the BOS boundary at heel-contact was lower when stepping was performed at a maximal speed (the RAPID condition in their study) as compared to a self-selected speed condition (PREF condition). This ML distance decreased even though step width increased, as in the present study. These authors further noted that the ML COM velocity at heel-contact decreased from the PREF to the RAPID condition whereas it did not 

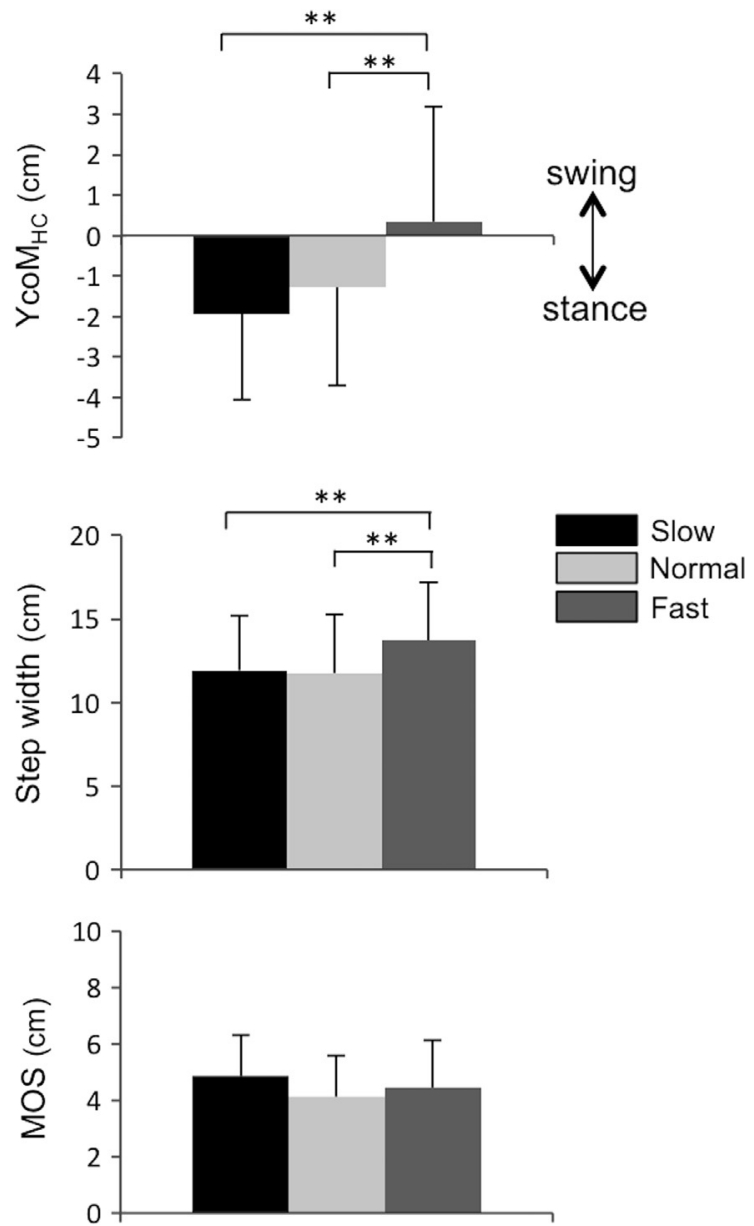

Fig. 5. Comparison of the mediolateral position of extrapolated centre of mass at swing heel-contact $\left(\mathrm{YcoM}_{\mathrm{HC}}\right)$, step width and margin of stability (MOS) under the slow, normal and fast speed conditions. Reported values are means \pm 1 standard deviation (all subjects combined). **: significant difference with $p<0.01$.

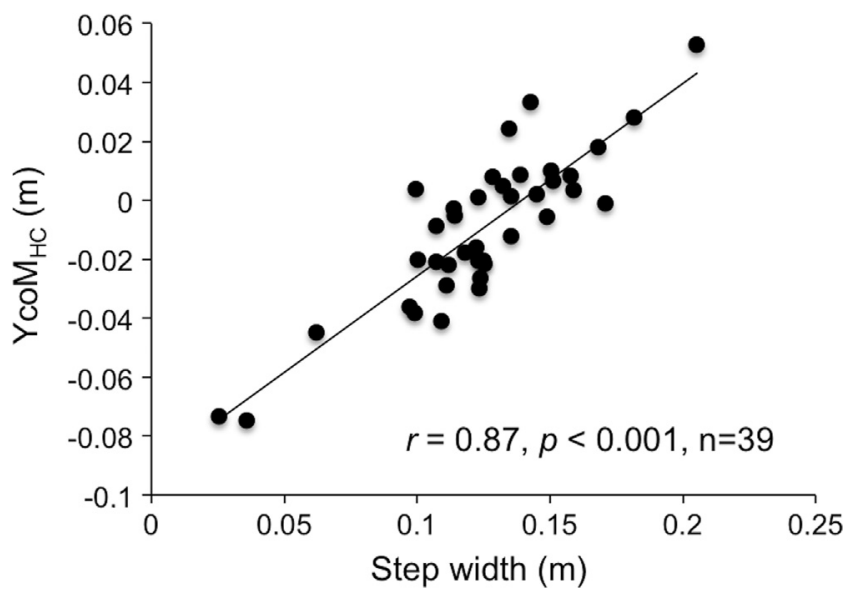

Fig. 6. Relationship between the mediolateral position of the extrapolated centre of mass at heel-contact $\left(\mathrm{YcoM}_{\mathrm{HC}}\right)$ and step width. Each point represents the mean of all trials performed in one speed condition for one subject.

change across the speed conditions in our study. The origin of this discrepancy between our results and the results of Singer et al. (2013) might stem from several experimental factors, e.g. the difference in the level of temporal pressure imposed on the rapid movement (self-initiated situation in the present study vs. reaction-time situation in Singer et al.'s study), the difference in the population tested (young adults vs. combined young and older adults), and the motor task itself (GI vs. single step). Besides the reasons for this discrepancy, it is worthwhile noting that the changes reported by Singer et al. (2013) in the COM dynamics at heel-contact between the PREF and RAPID condition are consistent with the hypothesis of an invariant MOS.

One limitation of the present study was that the various gait speeds were subjectively selected by the subjects, inducing nonhomogenous speed differences across the conditions. A smaller difference in the gait speed was observed between Slow and Normal ( $30 \pm 10 \%)$, compared to Normal and Fast ( $41 \pm 16 \%)$. This smaller speed difference could be responsible for the lesser variation of the dependant variables (e.g., $\mathrm{YcoM}_{\mathrm{HC}}$ and step width) obtained between Slow and Normal, compared to the difference observed between Normal and Fast.

In conclusion, the present study investigated the influence of gait speed on the control of mediolateral dynamic stability during gait initiation. Our results showed that when gait speed increased, mediolateral APA and step width were fine-tuned so as to maintain equivalent dynamic stability at the time of swing heel-contact. A decline in the control of mediolateral stability is known to be a major source of falling in frail subjects such as the elderly (Maki, 1997; Robinovitch et al., 2013). The approach used in the present study might be relevant to a better understanding of the aetiology of falls in these populations.

\section{Conflict of interest statement}

None of the authors have financial or other conflicts of interest in regards to this research.

\section{Acknowledgements}

This work was supported by a Grant from the University of La Reunion. The authors wish to thank Mr. Xavier Robert-Lachaine for his help during the experiment and Mr. Firmin P. Houte-Guelle for his comments on the manuscript.

\section{Appendix A. Supplementary material}

Supplementary data associated with this article can be found in the online version at http://dx.doi.org/10.1016/j.jbiomech.2013.11.011.

\section{References}

AMTI Model OR6-7 Biomechanics Platform Instruction Manual. Advanced Mechanical Technology, Inc. Watertown, MA, USA.

Azuma, T., Ito, T., Yamashita, N., 2007. Effects of changing the initial horizontal location of the center of mass on the anticipatory postural adjustments and task performance associated with step initiation. Gait Posture 26, 526-531.

Bouisset, S., Do, M.C., 2008. Posture, dynamic stability, and voluntary movement. Neurophysiol. Clin./Clin. Neurophysiol. 38, 345-362.

Brenière, Y, Do, M.C. 1986. When and how does steady state gait movement induced from upright posture begin? J. Biomech. 19, 1035-1040.

Brenière, Y., Do, M.C., Bouisset, S., 1987. Are dynamic phenomena prior to stepping essential to walking. J. Mot. Behav. 19, 62-76.

Caderby, T., Dalleau, G., Leroyer, P., Bonazzi, B., Chane-Teng, D., Do, M.C., 2013. Does an additional load modify the Anticipatory Postural Adjustments in gait initiation? Gait Posture 37, 144-146.

Corbeil, P., Anaka, E., 2011. Combined effects of speed and directional change on postural adjustments during gait initiation. J. Electromyogr. Kinesiol. 21, 734-741.

Ghoussayni, S., Stevens, C., Durham, S., Ewins, D., 2004. Assessment and validation of a simple automated method for the detection of gait events and intervals. Gait Posture 20, 266-272.

Hasson, C.J., Van Emmerik, R.E., Caldwell, G.E., 2008. Predicting dynamic postural instability using center of mass time-to-contact information. J. Biomech. 41, 2121-2129. 
Hof, A.L., Bockel, R.M.V., Schoppen, T., Postema, K., 2007. Control of lateral balance in walking experimental findings in normal subjects and above-knee amputees. Gait Posture 25, 250-258.

Hof, A.L., Gazendam, M.G., Sinke, W.E., 2005. The condition for dynamic stability. J. Biomech. 38, 1-8.

Ito, T., Azuma, T., Yamashita, N., 2003. Anticipatory control in the initiation of a single step under biomechanical constraints in humans. Neurosci. Lett. 352, 207-210.

Jian, Y., Winter, D.A., Ishac, M.G., Gilchrist, L., 1993. Trajectory of the body COG and COP during initiation and termination of gait. Gait Posture 1, 9-22.

Lepers, R., Brenière, Y., 1995. The role of anticipatory postural adjustments and gravity in gait initiation. Exp. Brain Res. 107, 118-124.

Lin, S.I., Yang, W.C., 2011. Effect of plantar desensitization on postural adjustments prior to step initiation. Gait Posture 34, 451-456.

Lyon, I.N., Day, B.L., 1997. Control of frontal plane body motion in human stepping. Exp. Brain Res. 115, 345-356.

Maki, B.E., 1997. Gait changes in older adults: predictors of falls or indicators of fear. J. Am. Geriatr. Soc. 45, 313-320.

McIlroy, W.E., Maki, B.E., 1999. The control of lateral stability during rapid stepping reactions evoked by antero-posterior perturbation: does anticipatory contro play a role? Gait Posture 9, 190-198.

McIlroy, W.E., Maki, B.E., 1997. Preferred placement of the feet during quiet stance: development of a standardized foot placement for balance testing. Clin. Biomech. (Bristol, Avon) 12, 66-70.

Menz, H.B., Lord, S.R., Fitzpatrick, R.C., 2003. Age-related differences in walking stability. Age Ageing 32, 137-142.

Mickelborough, J., Van Der Linden, M.L., Richards, J., Ennos, A.R., 2000. Validity and reliability of a kinematic protocol for determining foot contact events. Gait Posture 11, 32-37.

Polcyn, A.F., Lipsitz, L.A., Kerrigan, D.C., Collins, J.J., 1998. Age-related changes in the initiation of gait: degradation of central mechanisms for momentum generation. Arch. Phys. Med. Rehabil. 79, 1582-1589.

Robinovitch, S.N., Feldman, F., Yang, Y., Schonnop, R., Leung, P.M., Sarraf, T., SimsGould, J., Loughin, M., 2013. Video capture of the circumstances of falls in elderly people residing in long-term care: an observational study. Lancet 381, 47-54.

Rogers, M.W., Hedman, L.D., Johnson, M.E., Cain, T.D., Hanke, T.A., 2001. Lateral stability during forward-induced stepping for dynamic balance recovery in young and older adults. J. Gerontol. A: Biol. Sci. Med. Sci. 56, M589-594.

Rosenblatt, N.J., Grabiner, M.D., 2010. Measures of frontal plane stability during treadmill and overground walking. Gait Posture 31, 380-384.

Shkuratova, N., Morris, M.E., Huxham, F., 2004. Effects of age on balance control during walking. Arch. Phys. Med. Rehabil. 85, 582-588.

Singer, J.C., Prentice, S.D., McIlroy, W.E., 2013. Age-related changes in mediolateral dynamic stability control during volitional stepping. Gait Posture 38, 679-683.

Winter, D.A., 1995. A.B.C. (Anatomy, Biomechanics and Control) of balance during standing and walking. Waterloo Biomechanics, Waterloo.

Winter, D.A., 1990. Biomechanics and Motor Control of Human Movement, 2nd ed. Wiley, New York.

Yiou, E., Caderby, T., Hussein, T., 2012a. Adaptability of anticipatory postural adjustments associated with voluntary movement. World J. Orthop. 3, 75-86.

Yiou, E., Deroche, T., Do, M.C., Woodman, T., 2011. Influence of fear of falling on anticipatory postural control of medio-lateral stability during rapid leg flexion. Eur. J. Appl. Physiol. 111, 611-620.

Yiou, E., Do, M.C., 2011. Effects of medio-lateral postural perturbation induced by voluntary arm raising on the biomechanical organization of rapid step initiation. Mot. Control 15, 507-524.

Yiou, E., Hussein, T., Larue, J., 2012b. Influence of temporal pressure on anticipatory postural control of medio-lateral stability during rapid leg flexion. Gait Posture 35, 494-499.

Zettel, J.L., Mcllroy, W.E., Maki, B.E., 2002a. Can stabilizing features of rapid triggered stepping reactions be modulated to meet environmental constraints? Exp. Brain Res. 145, 297-308.

Zettel, J.L., Mcllroy, W.E., Maki, B.E., 2002b. Environmental constraints on foot trajectory reveal the capacity for modulation of anticipatory postural adjustments during rapid triggered stepping reactions. Exp. Brain Res. 146, 38-47. 